\title{
Conhecimento das Gestantes acerca do Parto na Admissão Intrapartal
}

\author{
Symara Abrantes Albuquerque de Oliveira Cabral ${ }^{1}$; Larissa Araújo do Carmo ${ }^{2}$; Sylvio Elvis da Silva Barbosa ${ }^{3}$; \\ Núbia Anuncia Silva de Fontes ${ }^{4}$;gor de Sousa Gabriel ${ }^{5}$; Marianna Leite Barroso ${ }^{6}$
}

\begin{abstract}
Resumo: O nascimento de uma criança é, sem dúvida, o evento mais importante na vida dos seres humanos, seja para quem estará gestando e parindo, seja para criança e mesmo para aqueles que os cercam. Neste sentido, a assistência é importante, especialmente quando ao desfecho do evento, ao parto. Deste modo o presente trabalho buscou, através de um estudo de campo avaliar a importância do conhecimento de gestantes acerca do parto no favorecimento para o desfecho gestacional típico. Deste modo, observou-se que no serviço há uma predominância de partos cesáreos, que as informações prestadas no pré-natal são insuficientes, e ainda que mesmo motivadas para o parto vaginal, os desfechos são, em sua maioria cesáreas sem indicações legítimas para tal. Diante dos resultados conclui-se que há a necessidade de revisitar práticas e muda-las de modo a buscar o devido respeito a mulher e o provimento das melhores estratégias de cuidado as pessoas envolvidas no evento do parto.
\end{abstract}

Palavras-chave: Cuidado Pré-Natal; Parto; Gestantes.

\section{Knowledge of Pregnant Women about Intrapartal Admission}

Abstract: The birth of a child is undoubtedly the most important event in the life of human beings, whether it be gestating and giving birth, whether it is for children or even for those around them. In this regard, care is important, especially when the outcome of the event, at delivery. In this way, the present study sought, through a field study, to evaluate the importance of the knowledge of pregnant women about delivery in favor of the typical gestational outcome. Thus, it was observed that in the service there is a predominance of cesarean deliveries, that the information provided in the prenatal period is insufficient, and even if motivated for vaginal delivery, the outcomes are mostly cesarean without legitimate indications for such. Given the results, it is concluded that there is a need to revisit practices and change them in order to seek due respect for women and providing the best care strategies to those involved in the delivery event.

Keywords: Prenatal Care; Childbirth; Pregnant women.

\footnotetext{
${ }^{1}$ Graduação em Enfermagem pela Universidade Federal de Campina Grande (2011). Especializações em: Saúde da Família, Obstetrícia, Gestão e Metodologias ativas. Mestrado multiprofissional em Sistemas Agroindustriais com ênfase na saúde. Cursando Doutorado em Ciências da Saúde pela Santa Casa da Misericórdia de São Paulo. Atuando principalmente nos seguintes temas: Enfermagem, Enfermagem em Saúde da Mulher, Ensino e aprendizagem, Metodologias Ativas de ensino e aprendizagem. Contato: .symara_abrantes@hotmail.com; ${ }^{2}$ Acadêmica do Curso de Graduação em Medicina pela Faculdade Santa Maria - FSM, Cajazeiras - PB. Possui Graduação em Enfermagem, pela Universidade Regional do Cariri - URCA (2012) e Curso Técnico em Informática pelo Instituto Federal do Ceará - IFCE;

${ }_{3}^{3}$ Acadêmico do Curso de Graduação em Medicina pela Faculdade Santa Maria (FSM), Cajazeiras - PB. Possui Graduação em Engenharia Metalúrgica pela Escola de Minas/Universidade Federal de Ouro Preto (2000). Mestre em Engenharia Ambiental - Uso e Conservação de Recursos Hídricos pela Universidade Federal de Ouro Preto (2003).;

${ }^{4}$ Universidade Federal de Campina Grande.

${ }^{5}$ Graduação em Medicina pela Universidade Federal da Paraíba (2007). Especialista em Medicina de Família e Comunidade com Residência médica pela Universidade Federal de Campina Grande (UFCG) - Campus Cajazeiras. Atualmente é médico de estratégia de saúde da família da Prefeitura Municipal de Cajazeiras e professor da Faculdade Santa Maria de Cajazeiras. Tem experiência na área de Saúde Coletiva, com ênfase em Medicina de Família e Comunidade

${ }^{6}$ Graduação em Enfermagem pela Faculdade de Enfermagem São Vicente de Paula (2010). Mestra em Saúde Pública, Diploma reconhecido pela UNIVERSIDADE FEDERAL DE PERNABUCO, dando os direitos e prerrogativas inerentes ao Diploma de MESTRA EM ENFERMAGEM no âmbito de todo território nacional. Atualmente é Enfermeira Programa de Saúde da Família da Prefeitura Municipal de Santa Helena, Enfermeira Obstétrica - Maternidade Dr Deodato Cartaxo e Docente - FASP - FACULDADE SAO FRANCISCO DA PARAIBA. Contato: mariannaleite_@hotmail.com.
} 


\section{Introdução}

Uma assistência qualificada e satisfatória, durante o pré-natal, o parto e o puerpério são fundamentais para garantir uma boa condição de saúde, tanto para a mulher quanto para o recém-nascido. Visando proporcionar à mulher uma experiência de vida gratificante, respeitando sua autonomia nas decisões em relação à sua saúde e garantindo o acesso a uma rede integrada de serviços, com adequada infraestrutura, que propicie abordagem integral do processo saúde/doença (CALIFE; LAGO; LAVRAS, 2010).

Essa assistência é iniciada no pré-natal, na qual os profissionais de saúde realizam consultas mensais, desenvolvendo procedimentos e condutas durante todo o ciclo gravídicopuerperal. Esse trabalho tem um papel importante na redução da morbimortalidade materna. É nesse momento, que a equipe de saúde da família, deve promover ações educativas com objetivo de informar e conscientizar as gestantes sobre os pródromos do trabalho de parto, bem como os tipos de parto e suas vantagens e desvantagens (CUNHA et al., 2009).

A escolha do tipo de parto, vaginal (normal) ou cirúrgico (cesárea), é assunto complexo e polêmico, mas diante da maneira como esse assunto for trabalhado durante o prénatal, será mais fácil conscientizar as gestantes que a cesariana, é um procedimento de exceção, indicada em situações de risco de vida para a gestante e/ou feto (MANDARINO et al., 2009).

Segundo a Organização Mundial de Saúde (OMS), o objetivo da assistência ao parto é manter mulheres e recém-nascidos sadios, com o mínimo de intervenções médicas, buscando garantir a segurança de ambos. Dessa maneira, a OMS recomenda que o profissional de saúde intervenha no nascimento de uma criança somente quando necessário, preconizando como ideal uma taxa de cesarianas entre $10 \%$ e 15\%, mas a incidência está aumentando em diversos países, como no Brasil, na qual as taxas são superiores a $35 \%$ em geral e ultrapassando os 70\%, quando se considera apenas o serviço privado (PATAH; MALIK, 2011).

Ainda segundo os autores acima, seria injusto responsabilizar apenas os médicos pelas elevadas taxas de cesárea, ignorando aspectos do relacionamento médico-paciente, bem como aspectos do contexto social e do atual modelo assistencial, público ou privado, de cada país 
Por isso, deve existir um trabalho nas Unidades Básicas de Saúde, de formar a proporcionar informações com o intuito de se preparar para o momento do nascimento, ao procurarem conhecer os tipos de parto, saber as vantagens do parto normal e da cesárea, ter informações sobre a fisiologia do parto normal e de que forma a via de parto pode influenciar na vida do seu filho (VELHO; SANTOS; COLLACO, 2014).

Neste sentido é que o presente estudo tem por objetivo avaliar a importância do conhecimento de gestantes acerca do parto no favorecimento para o desfecho gestacional típico. Além de: analisar os fatores condicionantes para escolha de via de parto; identificar potenciais informações que fragilizam ou fortalecem a mulher para o momento do parto; e qualificar o pré-natal quanto à sua atuação informativa das gestantes.

\section{Metodologia}

Trata-se de uma pesquisa de campo, exploratória, prospectiva, com abordagem quantitativa e qualitativa dos dados, realizada na Maternidade Dr. Deodato Cartaxo, do complexo hospitalar do Hospital Regional de Cajazeiras, localizado no município de Cajazeiras, que pertence à mesorregião do Sertão Paraibano e à microrregião de Cajazeiras e é o município-sede da Região Metropolitana de Cajazeiras, instituída pela lei complementar estadual $n^{\circ} 107$, de 8 de junho de 2013, que reúne quinze municípios da Paraíba, estando o serviço da maternidade regionalizado.

A população do estudo é constituída por todas as gestantes admitidas no serviço obstétrico de referência no período estabelecido para pesquisa, admitindo-se por critérios de inclusão: gestantes com idade gestacional a partir de 34 semanas em trabalho de parto ou pródomos admitidas no serviço e que se dispuserem a participar da pesquisa. Já como critérios excludentes tem-se as gestantes que não se enquadram nos critérios de inclusão e as que não aceitarem participar da pesquisa.

Foi utilizado como instrumento de coleta de dados o roteiro de entrevista semiestruturado, com dados qualitativos e quantitativos, sendo utilizada como metodologias de análise a estatística descritiva considerando um $\mathrm{p}<0,05$ e a análise de conteúdo proposta por Bardin (2009). 
Seguindo os preceitos éticos e científicos, tem-se o seguimento integral, sendo a pesquisa submetida ao comitê de ética e aprovada a partir de parecer sob número 2.092 .690 do Comitê de Ética em Pesquisa da Faculdade Santa Maria.

\section{Resultados e Discussões}

A pesquisa foi realizada no período de 01 de maio a 31 de junho, de modo que foram atendidas 383 gestantes, sendo que apenas 350 foram submetidas ao parto, sendo 64\% submetidas a cesárea e $36 \%$ ao parto vaginal, a amostra foi constituída de 50 (cinquenta) gestantes, de modo a contemplar populações iguais, sendo constituída, assim, de $50 \%$ de partos cesárias, e $50 \%$ partos vaginais.

Importante considerar que o alto índice de cesarianas observadas no serviço a recomendação da Organização Mundial de Saúde (OMS) que, para o Brasil, estaria situada entre $25 \%$ e $30 \%$. Importante considerar que, de acordo com a Portaria $\mathrm{n}^{\circ} 306$ de 28 de março de 2016 que aprova as Diretrizes de Atenção à Gestante: a operação cesariana, a taxa de cesariana no Brasil está em torno de $56 \%$.

Tomando por base a idade dos participantes, foram determinados os intervalos utilizando a regra de Sturges, de modo a ser representado na figura 01:

Figura 01: idade das participantes da pesquisa distribuídas a partir do tipo de parto

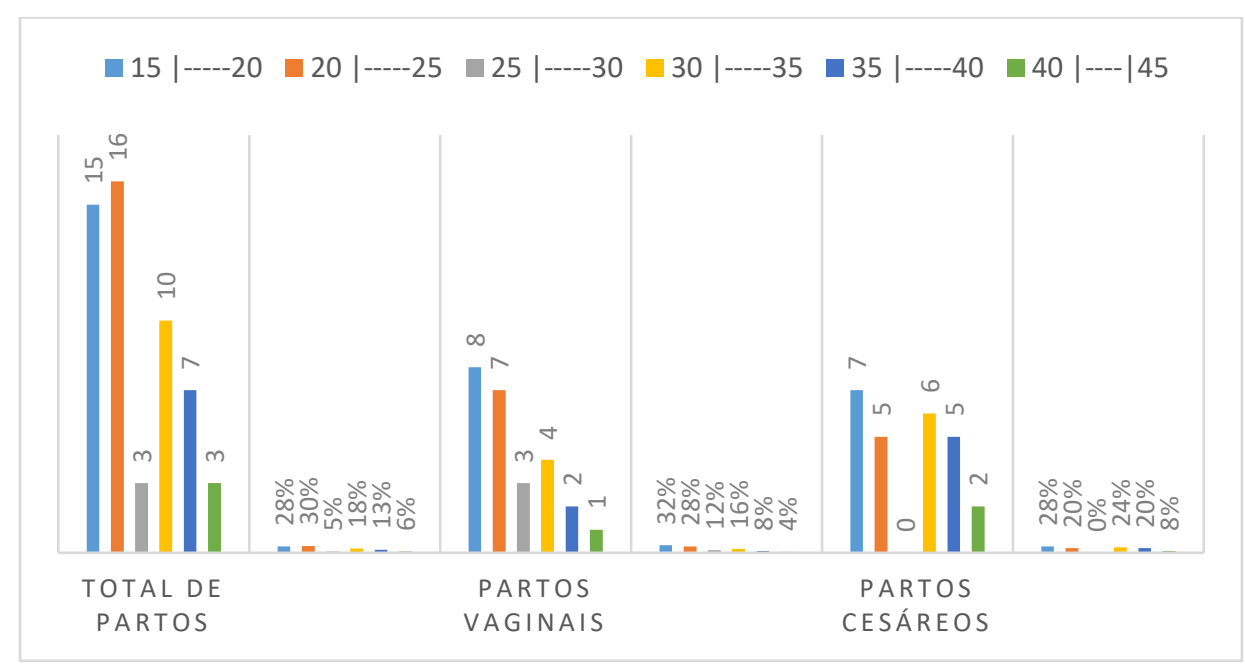

Fonte: dados da pesquisa, 2017. 
Os dados demonstraram que, considerando o total de participantes as participantes estão em uma média de idade de 25,5 anos, sendo que, para os partos vaginais 21 anos e para os cesáreos de 30 anos, considerando-se, deste modo, pode-se observar que, com a progressão da idade, há mais incidência de a gestante ser submetida a cesárea $(\mathrm{p}<0,001)$.

Considerando-se os antecedentes obstétricos

Figura 02: antecedentes obstétricos distribuídos a partir do tipo de parto $(\mathrm{p}<0,5)$.

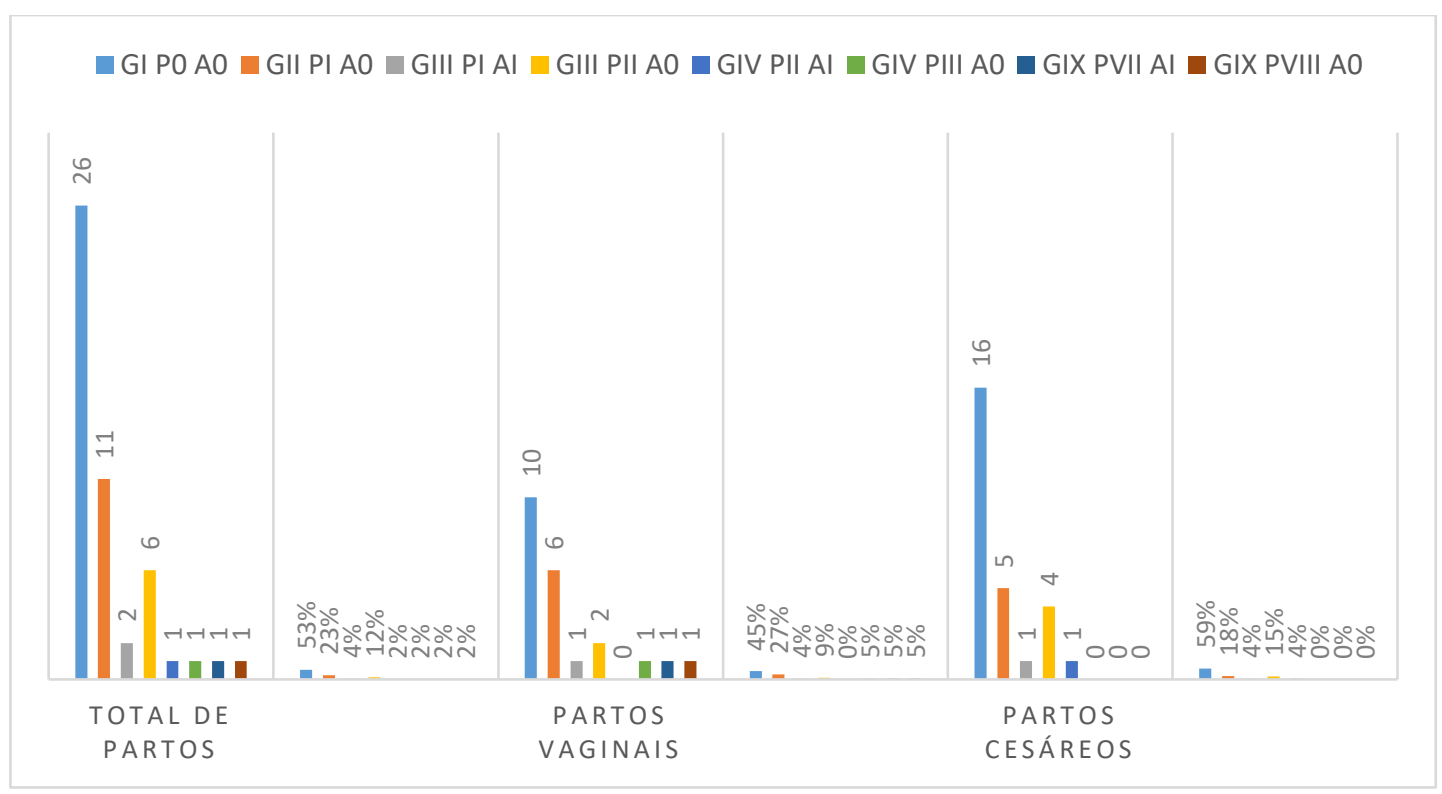

Fonte: dados da pesquisa, 2017.

De acordo com a figura 02 as nulíparas apresentaram maior incidência de partos cesáreos (59\%), ao tempo que observa-se maior paridade nas multíparas, não havendo no estudo paridade acima de 4 gestações, dados que correlacionam-se ao que Brasil (2016) revela, que "[...] as mulheres com três ou mais cesarianas prévias são relativamente raras, em nosso meio, uma vez que, no Brasil, é comumente realizada a ligadura tubária por ocasião da terceira cesariana”. Dados também observados no estudo, que revelou como situação no internamento o desejo pela laqueadura tubária (5\%). E ainda de acordo com a situação na internação, nenhuma delas revelou evidência para realização de parto cesáreo.

Observou-se ainda que nenhum parto natural aconteceu após cesárea anterior, contrariando evidências científicas que demonstram segurança no procedimento e, de acordo com Brasil (2016), deve-se ponderar o tipo de parto, correlacionando o risco de ruptura uterina ao risco aumentado de hemorragia, histerectomia, lesão vesical e de alças intestinais. 
Importante ainda considerar que "[...] a decisão em relação ao modo de nascimento considere o desejo e a autonomia feminina, obtendo-se um termo de consentimento informado".

Entendendo a importância do acompanhamento pré-natal para compreensão às inferências ou motivações e incentivos para o parto vaginal, observou-se um número médio de consultas de 8 para ambos os desfechos gestacionais, não incidindo para tal. Quando ao local do pré-natal 100\% o realizam na rede de Atenção Básica, 94\% exclusivamente na rede, e $6 \%$ na rede pública e privada $(\mathrm{p}<0,001)$.

Diante de tais dados, importante considerar que, na segunda metade do século passado, políticas públicas de saúde voltadas à mulher e à criança, foram instituídas no Brasil através do Programa Materno-Infantil (PMI), mediante as altas taxas de morbimortalidade sobre esses grupos. As ações contempladas no PMI, limitadas à assistência ao parto e ao acompanhamento da criança, vão ser ampliadas quase uma década depois, com a criação, no ano de 1984, do Programa de Assistência Integral à Saúde da Mulher (PAISM). Desde sua implantação, diversas ações voltadas ao ciclo gravídico puerperal foram sendo propostas e implementadas em busca de uma atenção de qualidade e de redução dos índices de morbimortalidade. Entre as diversas ações contempladas na atenção à mulher, a do pré-natal nos dias atuais é rediscutida abordando a concepção de saúde numa perspectiva integral, a de humanização como aporte teórico-filosófico norteador das práticas de saúde e a criação e a expansão da estratégia de saúde da família (COSTA et al., 2011).

No Brasil, o Ministério da Saúde, estabeleceu um protocolo com critérios mínimos a serem observados durante o acompanhamento pré-natal, garantido assistência de qualidade às gestantes com no mínimo 6 (seis) consultas de pré-natal. O Programa de Humanização no Pré-natal e Nascimento (PHPN) recomenda a realização de no mínimo seis consultas de prénatal para uma gestação a termo, com início do acompanhamento ainda no primeiro trimestre da gestação (8 a 12 semanas), para realização de algumas intervenções, tais como, a prevenção da transmissão vertical da sífilis e do HIV, diagnóstico de gravidez tubária, controle da anemia e manejo da hipertensão arterial e do diabetes, sendo fundamental a identificação precoce desses agravos. Além disso, seriam realizados exames laboratoriais e clínico-obstétricos somados às orientações sobre o período gravídico e puerperal (POLGLIANE et al., 2014). 
Tomando por base as dúvidas evidenciadas no pré-natal, os dados foram organizados e categorizados com base na análise de conteúdo e, de tal modo distribuídas por tipo de parto na figura 03 .

Figura 03: dúvidas no pré-natal distribuídas a partir do tipo de parto $(\mathrm{p}<0,5)$.

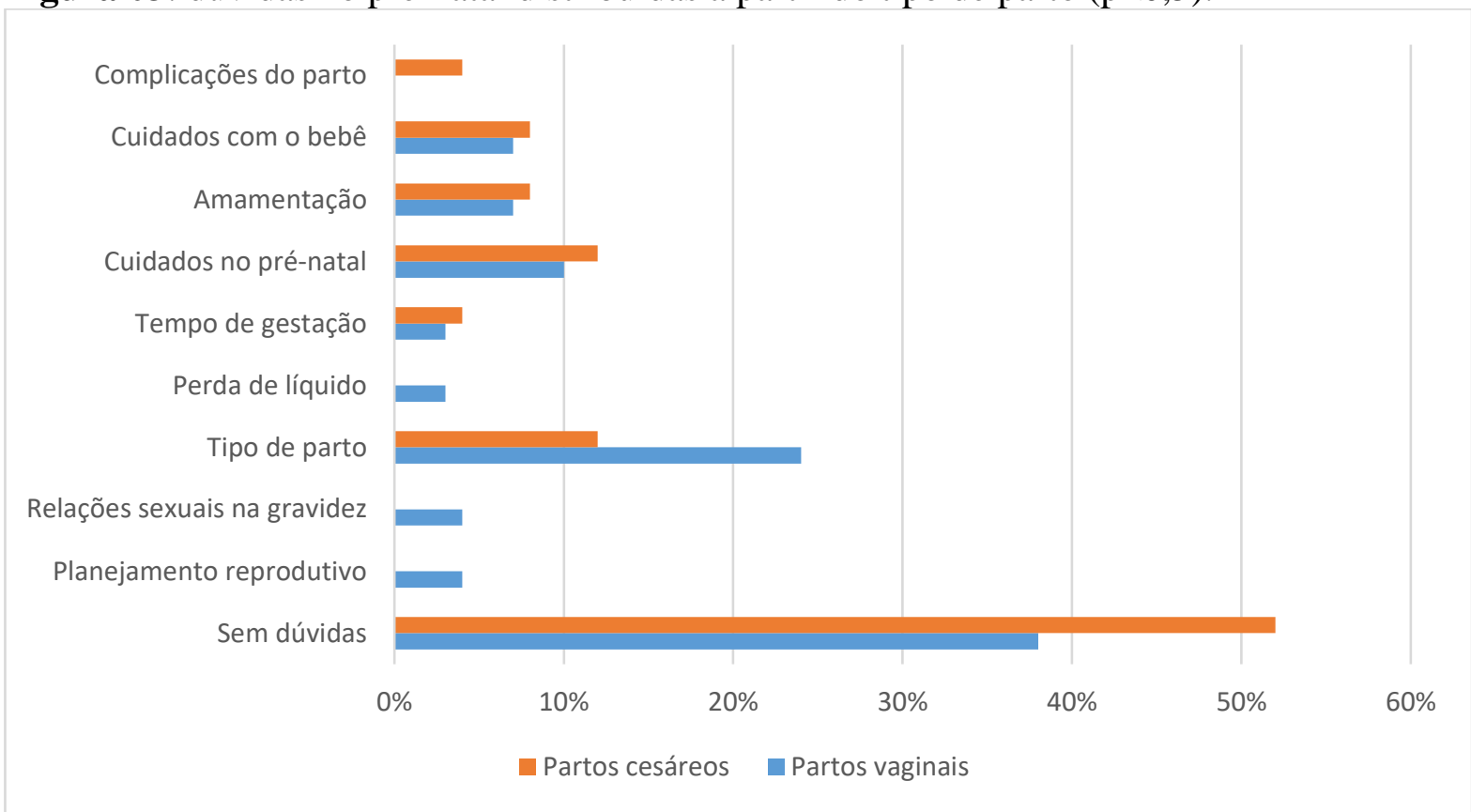

Fonte: dados da pesquisa, 2017.

Os dados demonstram que as mulheres submetidas a cesárea apresentaram menos dúvidas, especialmente quanto ao tipo de parto, possivelmente pela redução das expectativas frente ao enfrentamento do parto como um evento programado. O que não exclui a presença de preocupações quanto a complicações, exclusivamente presentes no parto cesáreo.

Quanto a resolução das dúvidas $41 \%$ das participantes relataram não ter suas dúvidas sanadas no momento do pré-natal, o que é preocupante, levando-se em consideração que durante a gravidez e o parto, as mulheres vivenciam várias alterações físicas e emocionais e expressam diversos valores e crenças, acerca do seu corpo e dos limites de sua autonomia para tomar decisões no processo de parturição. A escolha da mulher pelo parto normal ou cesáreo sofre algumas influências, como o papel da mulher no cenários sociocultural, relacionado a medicalização do corpo feminino, a compreensão familiar a respeito da gravidez, suas experiências e vivências anteriores e, também, o paradigma que informa o modo como é prestada a assistência em saúde e sua organização, que dependendo de como 
essa paciente é recebida e acompanhada no serviço de saúde, ela consegue desenvolver confiança, segurança e participação na escolha do seu tipo de parto (PIRES et al., 2010).

As expectativas das mulheres a respeito do tipo de parto, tem relação com o conhecimento das mesmas sobre o assunto e as informações que são tratadas pelos profissionais de saúde, durante o pré-natal, de forma a garantir uma atenção integral e de qualidade à mulher, esclarecendo suas dúvidas e anseios no que diz respeito aos aspectos da gestação, parto e puerpério. Sendo assim, é fundamental o apoio dos profissionais de saúde durante o período gestacional, subsidiando a escolha por meio da educação em saúde sobre essa temática (SANTANA; LAHM; SANTOS, 2015).

Quando as recomendações proferidas no pré-natal diversos foram os temas tratados, incluindo: alimentação saudável, repouso, realização de atividades físicas, cuidados com as mamas e amamentação, vacinação, prevenção de doenças como a dengue, cuidados com os bebês, realização de exames, medicamentos essenciais no pré-natal, e sobre os tipos de parto e recomendações puerperais.

O desenvolvimento de práticas educativas tanto nos espaços de atendimento individual, como através de trabalho de grupos, contribuem para o estabelecimento de práticas sociais, baseadas no diálogo e na troca de saberes, sendo uma forma de preparar a gestante para o parto e proporcionar autonomia para sua decidir entre parto vaginal ou cesariana (SOUZA; ROECKER; MARCON, 2011).

Nas ações desenvolvias durante o planejamento familiar e pré-concepcional, a mulher deverá ter a oportunidade de acesso à assistência pré-natal adequada e condições apropriadas para sua parturição. Sendo disponibilizados uma equipe de profissionais atuantes durante o trabalho de parto (enfermeira, fisioterapeutas, doulas e médicos obstetras) e um ambiente tipo PPP (pré-parto, parto e puerpério), para uma melhor assistência e conforto dessa paciente (REIS et al., 2014).

Nesse processo, deve ser elaborado um plano de parto, de forma a proporcionar informações sobre os procedimentos que serão realizados, o direito a um acompanhante de sua escolha e apoio dos profissionais de saúde da maternidade, contribuindo para seu bemestar e adequada evolução do trabalho de parto (SANTOS; OKAZAKI, 2012).

Essas orientações, abordadas durante o pré-natal, seriam relacionadas a promoção de comportamentos saudáveis, preparação e estímulo para o parto normal e aleitamento materno, e informações sobre os sinais de alerta de início do trabalho de parto. Também foi destacado 
em alguns trabalhos a importância de orientar sobre a maternidade de referência daquela gestante, reduzindo a peregrinação das mulheres no momento da admissão para o parto (DOMINGUES et al., 2015).

Neste sentido, as participantes foram questionadas quanto a via de parto desejada e quais orientações dadas no pré-natal, conforme dados descritos na figura 04.

Figura 04: via de parto de preferência distribuídas a partir do tipo de parto $(\mathrm{p}<0,001)$.

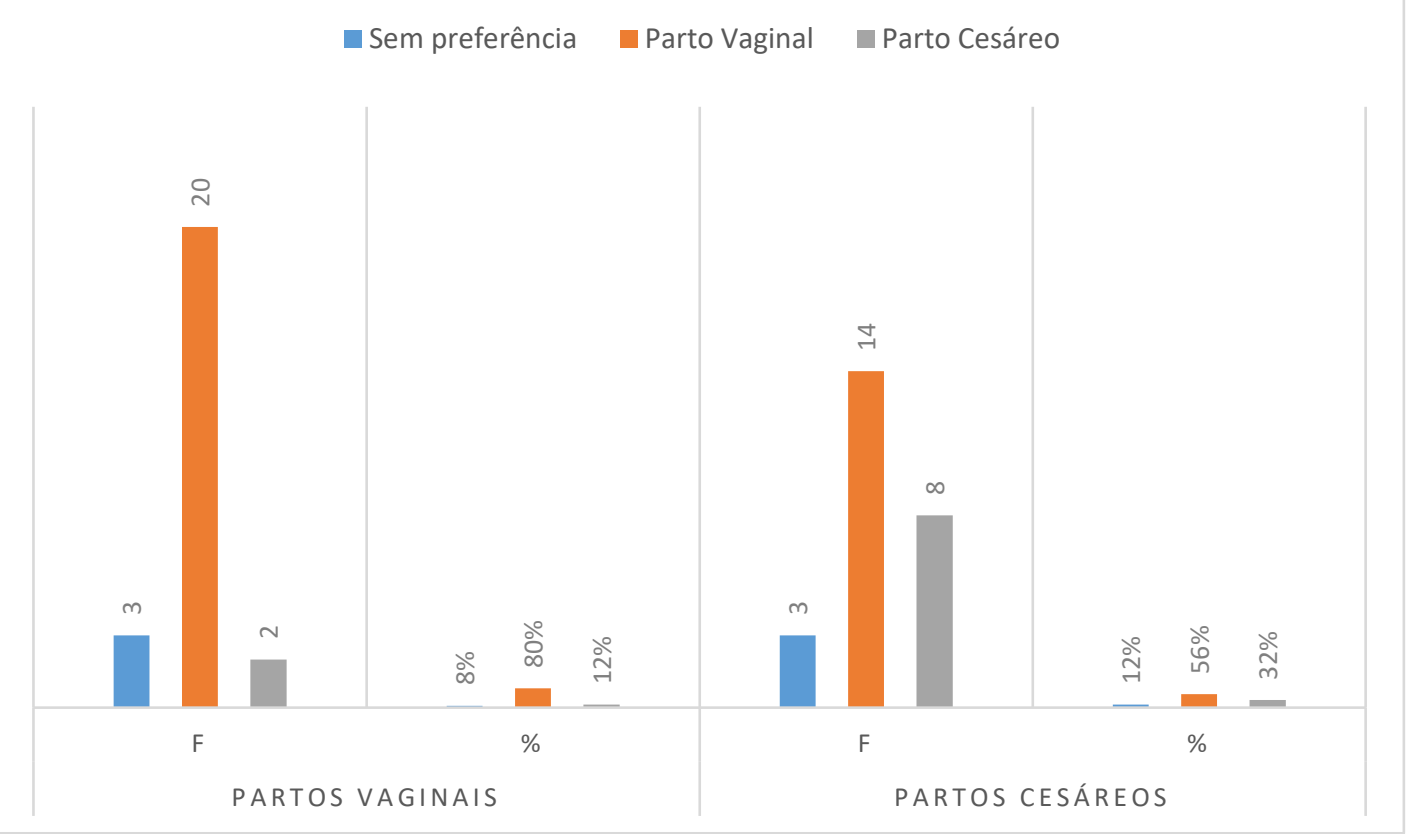

Fonte: dados da pesquisa, 2017.

Observa-se na figura 04 que as participantes que desejavam ter parto vaginal realmente o tiveram, de modo a entender-se que a motivação para o parto vaginal é fator determinante no desfecho da situação. Outro dado relevante diz respeito a motivação e desejo de algumas mulheres para o parto vaginal que tiveram desfechos de parto cesáreo, contrariando assim as suas próprias vontades, ao se correlacionar com o fato de que as situações iniciais de internação não condiziam com a necessidade de cesárea. Importante salientar que as pacientes com antecedentes obstétricos de parto vaginal que referiram desejar cesárea, o fizeram referindo o desejo pela laqueadura tubária.

Sobre as orientações dadas no pré-natal sobre a via de parto, observou-se que $40 \%$ referiram não ter recebido orientações, o que é preocupante, por definir déficit de informações sobre os benefícios do parto vaginal, podendo aumentar a ansiedade e influir no desfecho em 
parto cesáreo, e assim, quando questionadas de as orientações dadas mudaram o pensamento sobre a via de parto, $80 \%$ referiu não ter experimentado mudanças a partir das informações prestadas.

De acordo com Weidle et al. (2014) a maioria das gestantes preferem o parto vaginal; no entanto, a falta de atenção humanizada e a indução levam, muitas vezes, as mulheres a optarem pelo parto cesáreo. Além disso, o despreparo das mulheres para o parto vaginal interfere diretamente no sistema emocional da gestante ou parturiente, diminuindo a confiança dela na capacidade de ser protagonista do seu parto. Assim, ela não consegue perceber as vantagens do parto vaginal e conclui que cirurgia é melhor tanto para mãe quanto para o bebê. No entanto, a indicação correta de cesáreas traz vantagens, mas a indicação indiscriminada, sem critérios bem definidos, envolve riscos adicionais para mães e recém-nascidos.

A preferência pela via de parto pode variar do início para o final da gestação, vai depender das informações disponibilizadas durante o pré-natal e das medidas de humanização e acolhimento desenvolvidos pelos profissionais da maternidade, visando diminuir o medo e a ansiedade dessas pacientes (LEGUIZAMON JUNIOR; STEFFANI; BONAMIGO, 2013).

No entanto, o que se ver atualmente, são médicos promotores de uma cultura intervencionista por conveniência, justificando o ato cirúrgico pelo desejo das gestantes, principalmente as de classes sociais mais favorecidas, que preferem esta via para preservar a anatomia da genitália externa, para não sentir a dor do trabalho de parto e, entre aquelas com desejo de definir a prole, para aproveitar a ocasião e realizar a laqueadura tubária (esterilização cirúrgica) (MANDARINO et al., 2009).

O conceito de humanização da assistência ao parto inclui vários aspectos, alguns estão relacionados a mudança na cultura hospitalar, com a organização de uma assistência realmente voltada para as necessidades das gestantes, mudanças na estrutura física, construindo um ambiente mais acolhedor e favorável à implantação de práticas humanizadoras, e a atuação do profissional, respeitando os aspectos da fisiologia do parto, não intervindo desnecessariamente, e oferecendo suporte emocional à mulher e seus familiares (SANTOS; OKAZAKI, 2012).

No estudo realizado por Velho, Santos e Collaco (2014), o parto normal apresenta uma ambivalência de sentimentos relacionados as dificuldades enfrentadas no trabalho de parto, como sofrimento, dor e demora em sua evolução, em contraposição com as facilidades da recuperação e a possibilidade de adotar uma postura ativa, movimentar o seu corpo e 
realizar exercícios. Dentre as complicações foram citadas: a inflamação dos pontos, em caso de laceração ou episiotomia, febre e problemas relacionados à placenta. Já na cesárea, a dor está ausente inicialmente, mas aparece como uma consequência operatória, sendo mais persistente e incapacitante. Haverá maior risco de morbimortalidade materna e neonatal, de prematuridade e infecção, pode apresentar complicações como: cefaleia pós-raquianestesia, inflamação da ferida operatória, hemorragia, infecção puerperal, com necessidade de submeter-se a uma nova cirurgia, maior tempo de internação, necessidade de medicamentos e início tardio da amamentação.

Alguns médicos defendem o parto por via vaginal, por considerarem várias vantagens em relação à cesariana, como a recuperação é mais rápida e há menor chance de hematomas, infecções ou outras complicações cirúrgicas para a mãe. Porém, outros profissionais, afirmam que o parto por cesárea pode ser associado a efeito protetor para o neonato, por diminuição do risco de lesão do plexo braquial e de fraturas ósseas, e em relação à parturiente, menor risco de incontinência urinária e lesões perineais (CARDOSO; ALBERTI; PETROIANU, 2010)

$\mathrm{Na}$ assistência ao trabalho de parto normal, o obstetra deverá dispor de no mínimo, 6 a 10 horas, para acompanhar a evolução do trabalho de parto, principalmente em primíparas, na qual é mais prolongado, e realização de exame clínico periódico de 2 em 2 horas, para ausculta fetal, dinâmica uterina e toque vaginal. Na assistência realizada no serviço privado, o obstetra que, por diversos motivos, não puder disponibilizar desse tempo para a assistência, acabará indicando a cesariana. Já no serviço público, a gestante terá à sua disposição uma equipe de profissionais em regime de plantão, o que possibilita a continuidade do acompanhamento; assim, o motivo da realização das cesarianas estaria associado mais às reais indicações médicas (ameaça ao binômio materno-fetal) do que à "falta de tempo" (MANDARINO et al., 2009).

Apesar disso, alguns obstetras preferem o parto cesariano, tanto a pedido da gestante, quanto por vantagens para eles próprios, pois no parto normal, o profissional precisa abdicar das outras tarefas para dedicar várias horas ao atendimento da parturiente. Sendo assim, a demanda do parto cirúrgico é maior sustentada pela conveniência do médico, somado ao medo da dor, desinformação e receio da gestante, em relação as consequências do parto por via vaginal, considerando-o uma experiência arriscada (CARDOSO; ALBERTI; PETROIANU, 2010). 


\section{Considerações Finais}

Inquestionáveis são as vantagens do parto vaginal, e evidente é a influência das informações prestadas no pré-natal e da motivação da mulher para o parto vaginal, de modo que a promoção de orientações por profissionais de saúde, é fator determinante para a segurança e motivação.

Infelizmente, mesmo motivadas ao parto vaginal, muitas mulheres são submetidas a cesáreas desnecessárias, seja pela conveniência do serviço, dos profissionais ou mesmo pelo despreparo destes, que passam a considerar a impossibilidade do parto vaginal incoerentemente.

Conhecendo tal realidade torna-se evidente a necessidade de redirecionamento e revisão de práticas, seja pelo estabelecimento de protocolos nos serviços ou ainda pela capacitação dos profissionais, e sobretudo pelo respeito aos desejos, vontades e anseios das mulheres.

\section{Referências}

BARDIN, L. Análise de Conteúdo. Lisboa, Portugal; Edições 70, LDA, 2009.

BRASIL. PORTARIA N³06, DE 28 DE MARÇO DE 2016. Aprova as Diretrizes de Atenção à Gestante: a operação cesariana.

CALIFE, Karina; LAGO, Tania; LAVRAS, Carmen. Atenção à gestante e à puérpera no SUS - SP: manual técnico do pré natal e puerpério. Secretaria da Saúde. Coordenadoria de Planejamento em Saúde. Assessoria Técnica em Saúde da Mulher - São Paulo: SES/SP, 2010.

CARDOSO, Priscila Oliveira; ALBERTI, Luiz Ronaldo and PETROIANU, Andy.Morbidade neonatal e maternas relacionada ao tipo de parto. Ciênc. saúde coletiva [online]. $\quad 2010, \quad$ vol.15, n.2, pp.427-435. $\quad$ ISSN 1678 4561. http://dx.doi.org/10.1590/S1413-81232010000200019.

COSTA, ALEKSANDRA PEREIRA et al. CONTRIBUIÇÕES DO PRÉ-NATAL PARA O PARTO VAGINAL: PERCEPÇÃO DE PUÉRPERAS. Rev Rene, Fortaleza, 2011 jul/set; 12(3):548-54. 5 
CUNHA, Margarida de Aquino et al. Assistência pré-natal: competências essenciais desempenhadas por enfermeiros. Esc. Anna Nery [online]. 2009, vol.13, n.1, pp.145-153. ISSN 1414-8145. http://dx.doi.org/10.1590/S1414-81452009000100020.

DOMINGUES, Rosa Maria Soares Madeira et al.Adequação da assistência pré-natal segundo as características maternas no Brasil. Rev Panam Salud Publica [online]. 2015, vol.37, n.3, pp.140-147. ISSN 1680-5348.

LEGUIZAMON JUNIOR, Teodoro; STEFFANI, Jovani Antônio and BONAMIGO, Elcio Luiz.Escolha da via de parto: expectativa de gestantes e obstetras. Rev. Bioét. [online]. 2013, vol.21, n.3, pp.509-517. ISSN 1983-8042. http://dx.doi.org/10.1590/S198380422013000300015 .

MANDARINO, Natália Ribeiro et al.Aspectos relacionados à escolha do tipo de parto: um estudo comparativo entre uma maternidade pública e outra privada, em São Luís, Maranhão, Brasil. Cad. Saúde Pública [online]. 2009, vol.25, n.7, pp.1587-1596. ISSN 16784464. http://dx.doi.org/10.1590/S0102-311X2009000700017.

PATAH, Luciano Eduardo Maluf; MALIK, Ana Maria. Modelos de assistência ao parto e taxa de cesárea em diferentes países. Rev. Saúde Pública [online]. 2011, vol.45, n.1, pp.185194. ISSN 1518-8787. http://dx.doi.org/10.1590/S0034-89102011000100021.

PIRES, Denise et al.A influência da assistência profissional em saúde na escolha do tipo de parto: um olhar sócio antropológico na saúde suplementar brasileira. Rev. Bras. Saude Mater. Infant. [online]. 2010, vol.10, n.2, pp.191-197. ISSN 18069304. http://dx.doi.org/10.1590/S1519-38292010000200006.

POLGLIANE, Rúbia Bastos Soares et al.Adequação do processo de assistência pré-natal segundo critérios do Programa de Humanização do Pré-natal e Nascimento e da Organização Mundial de Saúde. Ciênc. saúde coletiva [online]. 2014, vol.19, n.7, pp.1999-2010. ISSN 1413-8123. http://dx.doi.org/10.1590/1413-81232014197.08622013.

REIS, Zilma Silveira Nogueira et al. Associação entre risco gestacional e tipo de parto com as repercussões maternas e neonatais. Rev Bras Ginecol Obstet. 2014; 36(2):65-71

SANTANA , Fernando Alves; LAHM , Janaína Verônica; SANTOS, Reginaldo Passoni dos. FATORES QUE INFLUENCIAM A GESTANTE NA ESCOLHA DO TIPO DE PARTO. Rev. Fac. Ciênc. Méd. Sorocaba, v. 17, n. 3, p. 123 - 127, 2015

SOUZA VB, ROECKER S, MARCON SS. Ações educativas durante a assistência pré-natal: percepção de gestantes atendidas na rede básica de Maringá-PR. Rev. Eletr. Enf. [Internet]. 2011 abr/jun;13(2):199-210. Available from: http://dx.doi.org/10.5216/ree.v13i2.10162.

VELHO, Manuela Beatriz; SANTOS, Evanguelia Kotzias Atherino dos and COLLACO, Vânia Sorgatto. Parto normal e cesárea: representações sociais de mulheres que os vivenciaram. Rev. bras. enferm. [online]. 2014, vol.67, n.2 [cited 2016-10-27], pp.282-289. Available from: $\quad<$ http://www.scielo.br/scielo.php?script=sci_arttext\&pid=S0034- 
71672014000200282\&lng=en\&nrm=iso>. ISSN 0034-7167. http://dx.doi.org/10.5935/00347167.20140038 .

WEIDLE, Welder Geison et al. Escolha da via de parto pela mulher: autonomia ou indução?. Cad. saúde colet. [online]. 2014, vol.22, n.1 [cited 2016-10-27], pp.46-53. Available from: $\quad<$ http://www.scielo.br/scielo.php?script=sci_arttext\&pid=S1414462X2014000100046\&lng=en\&nrm=iso $>\quad$ ISSN 462X. http://dx.doi.org/10.1590/1414-462X201400010008.

SANTOS, Isaqueline Sena; OKAZAKI, Egle de Lourdes Fontes Jardim. Assistência de enfermagem ao parto humanizado. Rev Enferm UNISA. 2012; 13(1): 64-8.

\section{Como citar este artigo (Formato ABNT):}

CABRAL, Symara Abrantes Albuquerque de Oliveira; CARMO, Larissa Araújo do; BARBOSA, Sylvio Elvis da Silva; FONTES, Núbia A. S. de; GABRIEL, Igor de Sousa; BARROSO, Marianna L. Conhecimento das Gestantes acerca do Parto na Admissão Intrapartal. Id on Line Revista ultidisciplinar e de Psicologia, 2018, vol.12, n.39, p.851-864. ISSN: 1981-1179.

Recebido: 31.01 .2018

Aceito: 01.02 .2018 